\title{
Evaluation of Malondialdehyde, Superoxide Dismutase and Catalase Activity in Fetal Cord Blood of Depressed Mothers
}

\author{
Mehmet Akif Camkurt ${ }^{1}$, Ebru Fındıklı ${ }^{2}$, Murat Bakacak ${ }^{3}$, Fatma İnanç Tolun ${ }^{4}$, Mehmet Fatih Karaaslan ${ }^{2}$ \\ ${ }^{1}$ Department of Psychiatry, Afşin State Hospital, Departments of ${ }^{2}$ Psychiatry, ${ }^{3}$ Obstetry, and ${ }^{4}$ Biochemistry, Faculty of Medicine, \\ Kahramanmaraş Sütçü İmam University, Kahramanmaraş, Turkey
}

\begin{abstract}
Objective: The umbilical cord consists of two arteries and one vein and it functions in the transport between the maternal and fetal circulation. Biochemical analysis of fetal cord blood (FCB) during delivery could be beneficial in terms of understanding the fetal environment. In this study, we aimed to investigate oxidative parameters like malondialdehyde (MDA), superoxide dismutase (SOD), and catalase (CAT) levels in FCB during delivery.

Methods: We collected FCB samples during caesarean section. Our study included 33 depressed mothers and 37 healthy controls. We investigated MDA, SOD, and CAT levels in FCB samples.

Results: We found no significant difference between groups in terms of MDA ( $p=0.625)$, SOD ( $p=0.940)$, and CAT ( $p=0.413)$ levels. Conclusion: Our study reveals probable protective effects of the placenta from oxidative stress. Future studies should include larger samples.
\end{abstract}

KEY WORDS: Fetal cord blood; Pregnancy; Depression; Malondialdehyde; Supeoxide dismutase; Catalase.

\section{INTRODUCTION}

The umbilical cord consists of two arteries and one vein. The umbilical vein functions in the transport of oxygenated and nutrient-rich blood to fetal circulation, and the umbilical arteries carry deoxygenated and nutrient-depleted blood from fetal circulation. Depression is a common psychiatric disorder, and it may get worse during pregnancy. ${ }^{1)}$ Moreover, depression imposes a burden on the mother, and could be related to worse birth outcomes, such as low birth weight, preterm birth, and intrauterine growth retardation. ${ }^{2,3)}$

Oxidative balance is the term used to define the equilibrium between oxidants and antioxidants. As revealed in several meta-analyses, oxidative stress is crucial in major depression. ${ }^{4-6)}$ Furthermore, there are several factors that form a basis for the vulnerability of the brain to oxidative stress. Relatively high oxygen consumption of the brain may be associated with an increase in free radicals. Lipid

\footnotetext{
Received: March 18, 2016 / Revised: April 21, 2016

Accepted: April 25, 2016

Address for correspondence: Mehmet Akif Camkurt, MD Department of Psychiatry, Afşin State Hospital, Yeşilyurt Mah., Kemal Ertekin Caddesi., 46500, Afşin/Kahramanmaraş, Turkey Tel: +90-506-440-44-00, Fax: +90-344-511-29-66 E-mail: dr.akif@gmail.com
}

tissue is a major component of the brain, which is a substrate for peroxidation. Likewise, excitatory neurotransmitters like glutamate exist in the brain. ${ }^{7)}$

As far as we know, malondialdehyde (MDA) is useful as an oxidative stress marker, and superoxide dismutase (SOD) and catalase (CAT) represent antioxidant enzymes. MDA is the final product of lipid peroxidation, and is frequently used to define oxidative stress. ${ }^{8)}$ In this context, several studies focused on MDA levels in psychiatric disorders. ${ }^{9)}$ SOD is the enzyme responsible for the conversion of superoxide anion radicals $\left(\mathrm{O}_{2} \bullet-\right)$ to hydrogen peroxide and molecular oxygen, and is important for controlling the cellular ROS levels. ${ }^{10)}$ For major depression, there are controversial reports for SOD activity, like decreased or increased activity. ${ }^{7)}$ The main function of CAT is the decomposition of hydrogen peroxide into water and oxygen, which prevents cells from developing oxidative stress. ${ }^{11)}$ To date, several studies reported similar CAT activity in major depression patients and healthy controls. ${ }^{12,13)}$

Oxidants and antioxidants may differ in different stages of pregnancy. In the earlier stages, the placental environment is hypoxic. As the number of mitochondria increases, the oxidative load shows increments. On the other hand, to compensate for the oxidative load, antioxidant mechanisms show a continuous increase. ${ }^{14)}$ 
Although it is a fact that stressful emotional conditions of mothers affect fetuses in a negative way, it is very invasive and complicated to investigate the effects of maternal conditions on fetal blood circulation before delivery. However, investigating biochemical parameters in fetal cord blood (FCB) during delivery could be a helpful approach for us to understand - for the last trimester - the intrauterine environment of the fetus. There are two studies in this regard. These studies point out a decrease in brainderived neurotrophic factor (BDNF) levels and an increase in tumor necrosis factor alpha (TNF- $\alpha)$ in FCB. ${ }^{15,16)}$ In this study, our aim is to investigate MDA, SOD, and CAT levels in the FCB of depressed and healthy mothers' babies.

\section{METHODS}

\section{Participants}

The participants were women giving birth by elective caesarean section at the Obstetric Clinic of Kahramanmaraş Sütçü İmam University (Kahramanmaraş, Turkey). The study sample included 33 women with a diagnosis of major depression alone according to the Diagnostic and Statistical Manual of Mental Disorders 4th edition and 37 women without any psychiatric diagnosis (controls) who met the study criteria. Our group studied total antioxidant status (TAS), total oxidant status (TOS), and oxidative stress index (OSI) levels in FCB of depressed mothers and healthy controls. ${ }^{5)}$ While TAS, TOS, and OSI represent external oxidant and antioxidant levels MDA, SOD, and CAT stand for body's own oxidant-antioxidant system. So, after investigating TAS, TOS, and OSI levels as external factors, in this study we aimed to investigate MDA, SOD, and CAT in terms of body's own oxidative-antioxidant parameters and we used the same serum samples (33 patients, 37 controls) which were collected in our previous research. ${ }^{5)}$ The inclusion criteria for the study as follows: voluntary participation to the study and current age between 18 and 40 years. Patients with a history of medical illnesses (e.g., endocrine abnormalities, cardiovascular and pulmonary system diseases, neurological disease, and metabolic disease), a history of pregnancy related complications, any malformation in newborn infants, a history of maternal infection, active maternal infections, mental retardation, multiple pregnancies, intrauterine growth restriction, low birth weight, preterm delivery or emergency caesarean section, those who reported smoking or alcohol consumption during pregnancy, who had used systemic corticosteroids during pregnancy, those that had used any psychotropic medications during pregnancy, and those in whose infants hypoxia had developed during delivery were excluded. The study was conducted in a similar methodology with previous studies. ${ }^{16)}$ Blood samples were collected from umbilical vein during the delivery, they were centrifuged at $4,000 \mathrm{rpm}$ for 10 minutes. Samples were stored at $-80^{\circ} \mathrm{C}$ until biochemical analysis.

\section{Biochemical Analysis}

The concentration of serum lipid peroxidation (total MDA) was determined by Ohkawa et al. ${ }^{17)}$ with slight modifications. MDA results were expressed in nanomoles per milliliter (nmol/ml). ${ }^{17)}$

CAT activity was assayed by measuring the degradation rate of $\mathrm{H}_{2} \mathrm{O}_{2}$ using Beutler's method. The rate of disappearance of $\mathrm{H}_{2} \mathrm{O}_{2}$ was monitored spectrophotometrically at $230 \mathrm{~nm}$. The assay medium consisted of $50 \mu 11 \mathrm{M}$ Tris HCI buffer ( $\mathrm{pH} 8$ ), $930 \mu \mathrm{l} 10 \mathrm{mM} \mathrm{H}_{2} \mathrm{O}_{2}, 930 \mu \mathrm{l}$ deionized water, and $20 \mu \mathrm{l}$ serum sample. One unit of CAT activity is defined as the amount of enzyme causing about $90 \%$ destruction of the substrate in 1 minute in a volume of $1 \mathrm{ml}$. CAT activity in the serum was expressed as $\mathrm{U} / \mathrm{ml}{ }^{18)}$

SOD activity was determined as described by Beyer and Fridovich. ${ }^{19)}$ This method employs xanthine and xanthine oxidase to generate superoxide radicals, which react with 2-(4-iodophenyl)-3-(4-nitro phenol-s-phenyl tetrazolium chloride) to form a red formazon dye. SOD activity is then measured by the degree of inhibition of this reaction.

\section{Statistical Analysis}

Statistical analysis was performed using the Statistical Package for Social Sciences, ver. 11.5 (SPSS, Inc., Chicago, IL, USA). The normality of continuous variables was assessed using the Shapiro-Wilk's W-test, histograms and steam-leaf graphs.

The chi-sqaure test used in comparison of categorical variables. While comparing normally distributed variables, Student's $t$-test was performed. The Mann-Whitney $U$-test was used to compare the two groups in comparison of non-parametric variables. Correlation coefficients and their significance were calculated with Spearman test (for non-normally distributed variables) and Pearson test (for normally distributed variables). In the current study we performed power analysis to evaluate adequacy of sample size. We found that power value for SOD was higher than 0.8 ; however, power values for MDA and CAT were between $0.5-0.8$. 


\section{RESULTS}

We didn't find any difference between groups regarding age (means of patients and controls were 29.6 and 31.5 years, respectively; $p=0.146)$. Furthermore, head circumference (mean of patients, $35.64 \mathrm{~cm}$; mean of controls, $35.81 \mathrm{~cm}$ ) and baby length (mean of patients, $50.82 \mathrm{~cm}$; mean of controls, $51.46 \mathrm{~cm}$ ) were similar in both groups. Beck Depression Scale scores of patients were significantly higher in patients while compared to controls ( $p$ $<0.001)$. Baby weight was significantly lower in patients than controls, 3,222 $\mathrm{g}$ and 3,490 g, respectively ( $p=0.004$ ) (Table 1). Patients and controls were similar in terms of vitamin supplementation ( $p=0.915)$.

MDA levels of patients and controls were similar, 14.60 $\mathrm{nmol} / \mathrm{ml}$ and $15.19 \mathrm{nmol} / \mathrm{ml}$, respectively $(p=0.625)$. Also we didn't find any difference between SOD levels in patients and controls, $3.439 \mathrm{U} / \mathrm{ml}$ and $3.411 \mathrm{U} / \mathrm{ml}$, respectively $(p=0.940)$. Moreover, CAT levels were similar in patients and controls, $57.5 \mathrm{U} / \mathrm{ml}$ and $52.0 \mathrm{U} / \mathrm{ml}$, respectively $(p=0.413)$ (Table 2$)$.

We find a weak correlation between birth weight and Beck-Depression Scale scores $(\mathrm{r}=-0.322, p=0.007)$. We didn't find any correlation between MDA, SOD, CAT levels and baby weight, head circumference and length.

\section{DISCUSSION}

In this study, we found no differences in MDA, SOD, and CAT levels in the FCB of depressed and healthy mothers' babies. However, we did find that the birth weights of babies were significantly lower in those whose mothers were depressed.

There is a delicate balance during pregnancy between oxidants and antioxidants. Although at the beginning of the pregnancy the placental environment is hypoxic, the increased number of mitochondria produces oxidants by oxygen consumption. Importantly, the fetal side of the placenta contains fewer lipid peroxides than the maternal side. ${ }^{14)}$ As oxidation increases, a compensatory increase should be observed in antioxidant levels. Consistently, free radical scavengers like SOD, bilirubin, and glutathione show continuous increments during the course of pregnancy. This response is considered to be compensatory. ${ }^{20)}$ FCB contains higher levels of antioxidants and a lower number of lipid peroxidation products. The conclusion of this study suggests that increased antioxidants are important for protecting the fetus against lipid peroxidation. ${ }^{21)}$

MDA has been shown to be increased in diverse groups of depressed patients. A study by Bilici et al. ${ }^{12)}$ revealed significant upregulation of MDA in depression patients.

Table 1. Clinical status of depressed mothers and healthy controls

\begin{tabular}{|c|c|c|c|c|}
\hline & Patient $(n=33)$ & Control $(n=37)$ & $z, \dagger$ & $p$ value \\
\hline Age $(y r)$ & $29.6 \pm 5.3$ & $31.5 \pm 5.5$ & $t=-1.469$ & 0.146 \\
\hline Baby weight (g) & $3,229 \pm 327$ & $3,490 \pm 404$ & $Z=-2.913$ & 0.004 \\
\hline Head circumference $(\mathrm{cm})$ & $35.64 \pm 1.22$ & $35.81 \pm 1.17$ & $Z=-0.626$ & 0.532 \\
\hline Length $(\mathrm{cm})$ & $50.82 \pm 1.59$ & $51.46 \pm 1.77$ & $Z=-1.508$ & 0.132 \\
\hline Vitamin supplement & & & & 0.915 \\
\hline Yes & $12(36.4)$ & $13(35.1)$ & & \\
\hline No & $21(63.6)$ & $24(64.9)$ & & \\
\hline Beck-D score & $16.9 \pm 8.99$ & $5.22 \pm 3.1$ & $Z=-5.724$ & $<0.001$ \\
\hline
\end{tabular}

Values are presented as mean \pm standard deviation or number (\%).

Groups were similar in terms of age. Birth weight of depressed mothers were significantly lower than healthy controls. Length of the baby and head circumference were similar in both groups.

Beck-D, Beck Depression Scale.

Table 2. MDA, SOD, and CAT levels in mothers

\begin{tabular}{|c|c|c|c|c|}
\hline \multirow{2}{*}{ Variable } & \multicolumn{2}{|c|}{ FCB } & \multirow{2}{*}{$z, \dagger$} & \multirow{2}{*}{$p$ value } \\
\hline & Depressed mother $(n=33)$ & Healthy mother $(n=37)$ & & \\
\hline MDA (nmol/ml) & $14.60 \pm 2.89$ & $15.19 \pm 3.46$ & $Z=-0.488$ & 0.625 \\
\hline $\mathrm{SOD}(\mathrm{U} / \mathrm{ml})$ & $3.439 \pm 1.563$ & $3.411 \pm 1.415$ & $t=0.076$ & 0.940 \\
\hline CAT (U/ml) & $57.5 \pm 24.1$ & $52.0 \pm 30.9$ & $t=0.823$ & 0.413 \\
\hline
\end{tabular}

We didn't find any significant difference between groups.

MDA, malondialdehyde; SOD, superoxide dismutase, CAT, catalase; FCB, fetal cord blood. 
Moreover, further studies reported that antidepressant treatment decreases MDA levels. Gałecki et al. ${ }^{13)}$ stated that MDA levels are significantly decreased following fluoxetine treatment, and Khanzode et $a l .{ }^{22)}$ found a decrease in MDA levels after selective serotonin reuptake inhibitor treatment. In our study, we found no difference between groups in terms of MDA levels. We consider that this may be due to the preventive effects of the placenta against oxidative stress. Furthermore, MDA may be unable to cross from maternal circulation to fetal circulation.

A growing body of evidence exists about SOD levels in depression. Red blood cell (RBC) SOD activity was higher in patients and decreased after treatment. ${ }^{23)}$ However, Herken $e t a l .{ }^{24)}$ found an increase in SOD levels after SSRI treatment. Gałecki et al. ${ }^{13)}$ pointed out that three months of treatment with fluoxetine did not alter RBC SOD activity. We found no difference in serum SOD levels. In the current study, some of the patients and controls were receiving vitamin supplements during caesarian sections. The similar results between groups may be due to vitamin intake. In this regard, mothers who take vitamins or iron supplements should be excluded in further studies.

The investigation of FCB is a novel methodological approach, and as far as we know, few studies have been performed in this context. TNF- $\alpha$ levels were investigated in mothers who have obsessive compulsive disorder. Results of that study pointed out that TNF- $\alpha$ levels were higher in the FCB of obsessive mothers. ${ }^{15)}$ The same group in another study, concluded that FCB BDNF levels were two-fold decreased in GAD patients. ${ }^{16)}$ We consider that maternal depression is not associated with an increase in MDA or a decrease in SOD and CAT in FCB. Future studies should evaluate MDA, SOD, and CAT levels in diverse psychiatric disorders.

Our study has some limitations. The methodological limitation is the collection of blood samples only from the umbilical vein. To overcome this issue, further studies should include FCB samples collected from both the umbilical vein and umbilical arteries. Furthermore, larger sample sized studies will be beneficial to overcome power weakness of our study.

In brief, MDA, SOD, and CAT levels were similar in the FCB of depressed mothers' babies and healthy controls. We consider that this similarity is due to preventive effects of the placenta against oxidative stress. Future studies should be done in larger samples and include blood samples collected simultaneously from the umbilical arteries and vein.

\section{REFERENCES}

1. Gotlib IH, Whiffen VE, Mount JH, Milne K, Cordy NI. Prevalence rates and demographic characteristics associated with depression in pregnancy and the postpartum. $J$ Consult Clin Psychol 1989:57:269-274.

2. Uguz F, Sahingoz M, Sonmez EO, Karsidag C, Yuksel G, Annagur $\mathrm{BB}$, et al. The effects of maternal major depression, generalized anxiety disorder, and panic disorder on birth weight and gestational age: a comparative study. $J$ Psychosom Res 2013;75:87-89.

3. Rondó PH, Ferreira RF, Nogueira F, Ribeiro MC, Lobert H, Artes R. Maternal psychological stress and distress as predictors of low birth weight, prematurity and intrauterine growth retardation. Eur J Clin Nutr 2003;57:266-272.

4. Black CN, Bot M, Scheffer PG, Cuijpers P, Penninx BW. Is depression associated with increased oxidative stress? A systematic review and meta-analysis. Psychoneuroendocrinology 2015;51:164-175.

5. Camkurt MA, Findiklı E, Tolun Fİ, Bakacak M, Bal NG, Sakall $\mathrm{H}$. Probable preventive effects of placenta from oxidative stress; Evaluation of total antioxidant status, total oxidant status and oxidative stress index in fetal cord blood during the delivery. Psychiatry Res 2016;240:222-225.

6. Palta P, Samuel LJ, Miller ER 3rd, Szanton SL. Depression and oxidative stress: results from a meta-analysis of observational studies. Psychosom Med 2014;76:12-19.

7. Ng F, Berk M, Dean O, Bush AI. Oxidative stress in psychiatric disorders: evidence base and therapeutic implications. Int J Neuropsychopharmacol 2008;11:851-876.

8. Bulut M, Selek S, Gergerlioglu HS, Savas HA, Yilmaz HR, Yuce $\mathrm{M}$, et al. Malondialdehyde levels in adult attentiondeficit hyperactivity disorder. J Psychiatry Neurosci 2007; 32:435-438

9. Lopresti AL, Maker GL, Hood SD, Drummond PD. A review of peripheral biomarkers in major depression: the potential of inflammatory and oxidative stress biomarkers. Prog Neuropsychopharmacol Biol Psychiatry 2014;48:102111.

10. Perry JJ, Shin DS, Getzoff ED, Tainer JA. The structural biochemistry of the superoxide dismutases. Biochim Biophys Acta 2010;1804:245-262.

11. Beers RF Jr, Sizer IW. A spectrophotometric method for measuring the breakdown of hydrogen peroxide by catalase. J Biol Chem 1952;195:133-140.

12. Bilici M, Efe H, Köroğlu MA, Uydu HA, Bekaroğlu M, Değer O. Antioxidative enzyme activities and lipid peroxidation in major depression: alterations by antidepressant treatments. J Affect Disord 2001;64:43-51.

13. Gałecki P, Szemraj J, Bieńkiewicz M, Florkowski A, Gałecka E. Lipid peroxidation and antioxidant protection in patients during acute depressive episodes and in remission after fluoxetine treatment. Pharmacol Rep 2009;61:436-447.

14. Casanueva E, Viteri FE. Iron and oxidative stress in pregnancy. J Nutr 2003;133(5 Suppl 2):1700S-1708S.

15. Uguz F, Onder Sonmez E, Sahingoz M, Gokmen Z, Basaran M, Gezginc K, et al. Neuroinflammation in the fetus exposed to maternal obsessive-compulsive disorder during pregnancy: a comparative study on cord blood tumor necrosis factor-alpha levels. Compr Psychiatry 2014;55:861-865.

16. Uguz F, Sonmez EO, Sahingoz M, Gokmen Z, Basaran M, Gezginc K, et al. Maternal generalized anxiety disorder during pregnancy and fetal brain development: a comparative study on cord blood brain-derived neurotrophic factor levels. J Psychosom Res 2013;75:346-350. 
17. Ohkawa H, Ohishi N, Yagi K. Assay for lipid peroxides in animal tissues by thiobarbituric acid reaction. Anal Biochem 1979;95:351-358.

18. Beutler E. Red cell metabolism: a manual of biochemical methods. New York, NY:Grune \& Stratton;1984.

19. Beyer WF Jr, Fridovich I. Assaying for superoxide dismutase activity: some large consequences of minor changes in conditions. Anal Biochem 1987;161:559-566.

20. Watson AL, Palmer ME, Jauniaux E, Burton GJ. Variations in expression of copper/zinc superoxide dismutase in villous trophoblast of the human placenta with gestational age. Placenta 1997;18:295-299.

21. Kiely M, Morrissey PA, Cogan PF, Kearney PJ. Low molecular weight plasma antioxidants and lipid peroxidation in maternal and cord blood. Eur J Clin Nutr 1999;53:
861-864.

22. Khanzode SD, Dakhale GN, Khanzode SS, Saoji A, Palasodkar R. Oxidative damage and major depression: the potential antioxidant action of selective serotonin re-uptake inhibitors. Redox Rep 2003;8:365-370.

23. Kotan VO, Sarandol E, Kirhan E, Ozkaya G, Kirli S. Effects of long-term antidepressant treatment on oxidative status in major depressive disorder: a 24-week follow-up study. Prog Neuropsychopharmacol Biol Psychiatry 2011;35:1284-1290.

24. Herken H, Gurel A, Selek S, Armutcu F, Ozen ME, Bulut $\mathrm{M}$, et al. Adenosine deaminase, nitric oxide, superoxide dismutase, and xanthine oxidase in patients with major depression: impact of antidepressant treatment. Arch Med Res 2007;38:247-252. 